\title{
Regular and Chaotic Orbits in a Self-consistent Triaxial Stellar System with Slow Figure Rotation
}

\author{
J. C. Muzzio (jcmuzzio@fcaglp.unlp.edu.ar) \\ Facultad de Ciencias Astronómicas y Geofísicas, Universidad Nacional de La \\ Plata, and Instituto de Astrofísica La Plata (UNLP-CONICET)
}

\begin{abstract}
We created a self-consistent triaxial stellar system through the cold disipationless collapse of 100,000 particles whose evolution was followed with a multipolar code. The resulting system rotates slowly even though its total angular momentum is zero, i.e., it offers an example of figure rotation. The potential of the system was subsequently approximated with interpolating formulae yielding a smooth potential stationary in the rotating frame. The Lyapunov exponents could then be computed for a randomly selected sample of 3,472 of the bodies that make up the system, allowing the recognition of regular and partially and fully chaotic orbits. The regular orbits were Fourier analyzed and classified using their locations on the frequency map. A comparison with a similar non-rotating model showed that the fraction of chaotic orbits is slightly but significantly enhanced in the rotating model; alternatively, there are no significant differences between the corresponding fractions neither of partially and fully chaotic orbits nor of long axis tubes, short axis tubes, boxes and boxlets among the regular orbits. This is a reasonable result because the rotation causes a breaking of the symmetry that may increase chaotic effects, but the rotation velocity is probably too small to produce any other significant differences. The increase in the fraction of chaotic orbits in the rotating system seems to be due mainly to the effect of the Coriolis force, rather than the centrifugal force, in good agreement with the results of other investigations.
\end{abstract}

Keywords: Triaxial stellar systems, figure rotation, stellar orbits, chaotic motion

\section{Introduction}

While it is relatively simple to build up models of self-consistent spherical and disk-like stellar systems, for which even analytical solutions are available, the problem is much more complicated in the case of triaxial stellar systems (see, e.g., Binney and Tremaine, 1987 and Merritt, 1999). The method of Schwarzschild (1979) is probably the most popular one among those used to investigate triaxial stellar systems, while the one pionnered by Sparke and Sellwood (1987) is being increasingly used in recent years. Using the latter approach, Muzzio et al. (2005) employed a quadrupolar N-body code to follow the disipationless collapse of an initially cold spherical distribution; once the equilibrium had been reached and the most energetic particles had been eliminated, they froze and smoothed the potential with an interpolating function

(C) 2006 Kluwer Academic Publishers. Printed in the Netherlands.

mine01cor.tex; 7/05/2006; 17:59; p.1 
which allowed them to obtain the orbits and Lyapunov exponents of a sample of the bodies that made up the system.

More recently, we followed the evolution of the system of Muzzio et al. over a much longer time interval than they have done and we found that, instead of being stationary as we had though, it rotates very slowly around its minor axis; moreover, as its total angular momentum is zero, this system is an interesting example of figure rotation, a phenomenon exhibited for example by the Riemann ellipsoids, in the case of fluids, and by the Freeman bars, in the case of stellar systems (see, e.g., Binney and Tremaine, 1987). Although the rotational velocity is very small, we deemed advisable to repeat our previous investigation taking into account the rotation to check whether any significant differences might arise. Besides, the frequency analysis method we had used to classify the regular orbits in our previous work failed to classify a substantial fraction of them but, more recently, Kalapotharakos and Voglis (2005) were able to classify all their orbits in similar triaxial systems using a somewhat different frequency analysis method; therefore, here we slightly improved their method and applied it both to our previous, non-rotating, system and to the present, rotating, one.

As the stellar system and most of our methods have been extensively described before, they are only briefly summarized in the next section and more detailed explanations are limited to the aspects that are new, i.e., the rotation of the system and the frequency analysis method. Our results are presented in the third section and discussed in the fourth one.

\section{Model and methods}

\subsection{Rotating tRiaXial STELlaR SYStem}

Here we use the same model stellar system already employed by Muzzio et al. (2005), which was created by the collisionless collapse of an initial spherical distribution of 100,000 particles with very low velocity dispersion. The collapse was followed using a version of the quadrupolar code of White (1983) kindly provided by L.A. Aguilar. The most energetic particles were discarded, to finally obtain a virialized selfconsistent stellar system of 86,818 particles; as shown in Table I and Fig. 2 of Muzzio et al., the system is strongly triaxial, with axial ratios decreasing towards its center, and its projected surface density is well adjusted by a de Vaucouleurs law. Our units are such that the initial mass of the system and the gravitational constant, $G$, are both equal to 1 (i.e., the final mass is thus 0.868). 
As the system virialized quickly, we had only followed it for about 25 time units (t.u. hereafter) after it had reached its triaxial shape in our original investigation. Nevertheless, more recently and for another study, we let it evolve for a few hundred time units and we noticed that it was not stationary as we had thought, but it rotated very slowly around its minor axis. As multipolar codes do not automatically conserve total linear and angular momenta, as direct summation codes do, we assured their conservation introducing the following improvements in the code: 1) Rather than using a heavier particle as the center of the expansion, we determined that center following the recipe of Cruz et al. (2002); 2) The sum of the forces over all the particles was made equal to zero at every integration step; 3) The sum of the torques over all the particles was also made equal to zero at every integration step. In this way, the initial zero angular momentum raised to only $10^{-4}$ at the end of the integration, so that we can feel confident that the observed rotation is indeed figure rotation.

We started with the final, virialized, system of Muzzio et al. (2005) and let it evolve for 600 t.u.; despite the much longer time interval used here the energy was conserved to $0.1 \%$, as in our previous work, thanks to the improvements introduced in the code. A plot of the angle between the direction of the major axis and its original direction was then used to derive the angular velocity. The plot was slightly curved, an effect probably due to the gradual expansion of the system due to relaxation effects that are present even in multipolar codes (see, e.g., Hernquist and Barnes, 1990); therefore, as we wanted to use the same system of our previous investigation, i.e., the initial one of the present study, we obtained the angular velocity from the tangent to the plot at its initial point. Its value turned out to be $-0.00975(t . u .)^{-1}$, which is indeed very low: it corresponds to a whole revolution in 645 t.u. while, as indicated by Muzzio et al., the Hubble time for a system like ours is of the order of between 115 and 575 t.u.; another way to see the same is noting that the corresponding corotation radius is about 20 units of length, while the bulk of the system lies within a radius of 2 or 3 of the same units. NGC 4365 might offer an example of a true stellar system with such a slow figure rotation, because Statler et al. (2004) found some evidence for a period of figure rotation about one-third the Hubble time and that periods ten times shorter could probably be ruled out.

An additional check was perfomed using S.J. Aarseth's NBODY2 direct summation code (e.g., Aarseth, 2003) but, since it is much slower than the quadrupolar code, only one-fourth of the particles (selected at random and with their masses multiplied by four) were included and the evolution was followed over one-tenth of the time interval only. 
The results obtained with this limited sample and time interval agreed very well with those of the quadrupolar code, showing that the figure rotation is real and independent of the code used.

The potential was approximated with the same equations and with the same values of their parameters used by Muzzio et al. (2005), the only difference being that now it rotates around its minor axis with a rotation velocity of $-0.00975(t . u .)^{-1}$. Just as we did in that study, we checked that the system remained stationary in the potential so represented by letting the system evolve in this potential, combining the results obtained at different times and computing the mean square errors that resulted from fitting the interpolating functions to these combined results. The new errors were essentially the same as those of Table II of Muzzio et al., so that we can conclude that the system also remains stationary in the rotating potential.

Those results provide another way of seeing how small the dynamical effect of the rotation actually is. Adopting the potential of the non-rotating system (as defined by the corresponding interpolating equation) as the basic one, we also considered the potentials resulting from the parameters obtained from the fittings used for Table II of Muzzio et al. at the initial time, $10 T_{\text {cr }}$ and $20 T_{\text {cr }}$ and at the initial time, $200 T_{\text {cr }}$ and $400 T_{\text {cr. }}$. The square root of the mean square percentual differences between the original and the new potentials, evaluated at the positions of the $N$-bodies, are $0.06 \%$ and $0.11 \%$, respectively, for the $0-10-20 T_{\text {cr }}$ and $0-200-400 T_{\text {cr }}$ cases. Alternatively, the square root of the mean square percentual difference between the original and the rotating potential, evaluated at the positions of the $N$-bodies is only $0.02 \%$. Two points are worth noting, however: 1 ) The differences in the potential arising from the motion of the particles in the nonrotating system would be much smaller in a real galaxy, because the number of its stars would be much larger than that of the bodies in the former; 2) While those differences are of the same order at all radii, the differences between the potentials of the non-rotating and rotating systems are strongly dependent on radius, going from zero at the center to $0.50 \%$ at the border of the system. We will see below how these differences may affect the orbital structure of the system.

\subsection{LYAPUNOV EXPONENTS}

Just as we had done in our previous investigation (Muzzio et al., 2005), finite time Lyapunov characteristic numbers (FT-LCNs hereafter) were computed using the LIAMAG routine (Udry and Pfenniger, 1988), kindly provided by D. Pfenniger. We computed the orbits from the same 3,472 initial positions and velocities used before, which had been 
randomly chosen among those of the bodies that make up our stellar system. The integration and normalization intervals were taken again, respectively, as 10,000 and 1 t.u., and the requested precision was $10^{-15}$; energy was conserved to better than $10^{-12}$ and the limiting value to separate zero from non-zero values of the FT-LCNs was again 0.00155 $(t . u .)^{-1}$.

Muzzio (2003), Muzzio and Mosquera (2004) and Muzzio et al. (2005) have shown that there are significant differences between the spatial distributions of bodies on partially and fully chaotic orbits, those on the former having distributions more akin to those of bodies on regular orbits. Therefore, following those authors, we used the largest $\left(L_{\max }\right.$ hereafter) and the second largest $\left(L_{\text {int }}\right.$ hereafter) FTLCNs to recognize regular $\left(L_{\max }<0.00155(t . u .)^{-1}\right)$, partially chaotic $\left(L_{i n t}<0.00155(t . u .)^{-1} \leq L_{\max }\right)$ and fully chaotic orbits (both $L_{\max }$ and $L_{\text {int }}$ larger than, or equal to, $0.00155(\text { t.u. })^{-1}$ ) in our sample.

\subsection{FREQUENCY ANALYSIS}

Muzzio et al. (2005) classified the regular orbits using the code of Carpintero and Aguilar (1998) which is based on the work of Binney and Spergel (1982) and Binney and Spergel (1984), but 15.3\% of those orbits could not be classified with that code. More recently, Kalapotharakos and Voglis (2005) could investigate their whole sample of orbits, in triaxial systems similar to ours, using the frequency map analysis pionnered by Papaphilippou and Laskar (1998). Therefore, we decided to repeat our previous investigation of the regular orbits in the non-rotating system and to perform the present one in the rotating system using the frequency map.

The modified Fourier transform code of S̆idlichovský and Nesvorný (1997) was kindly provided by D. Nesvorný and used for our analysis. Our $x, y$ and $z$ axes are oriented, respectively, as the major, intermediate and minor axes of our system, and the corresponding velocities are $u, v$ and $w$. The analysis to obtain the fundamental frequencies $f_{x}, f_{y}$ and $f_{z}$ was performed, respectively, on the complex variables $x+i u, y+i v$ and $z+i w$; these were derived from 8,192 points equally spaced in time obtained integrating the regular orbits over 300 radial periods. We used simple combinations of sines and cosines to check that, in this way, frequencies could be recovered with a precision better than $10^{-9}$. Similar precisions were attained for most of our orbits, judging from the values of positive and negative frequencies and the accuracy of the resonance relations. Nevertheless, the precision was much lower in many other cases and our experiments with combinations of sines and cosines showed that the presence of nearby lines could easily reduce 
the precision by several orders of magnitude. Comparisons between the positive and negative values of the same frequencies for our orbits and examination of the resonant lines and their surroundings in the frequency plane suggested a practical limit of $2 \times 10^{-4}$ for the precision, and that is the value we adopted.

Following Papaphilippou and Laskar (1998), Wachlin and FerrazMello (1998), and Kalapotharakos and Voglis (2005) we initially selected the frequency corresponding to the largest amplitude for each direction as the fundamental frequency of that direction. Nevertheless, we found that about $4 \%$ of the regular orbits had $f_{x} / f_{z}$ or $f_{y} / f_{z}$ values larger than 1.0, when one would expect that $f_{x} \leq f_{y} \leq f_{z}$. Besides, about $0.25 \%$ of the regular orbits, all of them short axis tubes (SATs hereafter), had $f_{x}$ and $f_{y}$ values lower than those corresponding to similar orbits of the same energy and appeared well detached, to the left and below, the other SATs on the frequency map. Both effects can be easily explained by the analysis of Binney and Spergel (1982). The former is due to libration and can be corrected as follows (Carpintero and Aguilar, 1998): when $f_{y}<f_{x}$ one has to check whether there is a pair of large amplitude $y$ lines symmetric with respect to $f_{x}$, in which case the frequency of the pair member with the largest (in absolute value) $f_{y}$ is the corresponding fundamental frequency; the same procedure is followed when $f_{z}<f_{y}$ or $f_{z}<f_{x}$. The latter effect is equivalent to having an epicycle larger than the closed orbit that supports it and it can be corrected taking as the fundamental frequency that with the second largest amplitude, provided that that amplitude is similar to the largest one and that the former frequency is larger than the latter one. All the orbits that needed these special treatments were low energy (in absolute value) orbits and, conversely, all the orbits with the lowest energies needed them. In the case of the SATs, the true cause is probably not so much the low energy as the fact that these low energy SATs are also extremely elongated ones, as a result of having created the model through a cold collapse: they reach minimum radii which are only $2 \%$ or $3 \%$ of their maximum radii.

Orbit 2768 in the non-rotating system is a good example of both effects and Table I presents a simplified version of its frequency analysis (we present less digits than the 16 typical of the double precision employed, we only show the 6 lines with the largest amplitudes, rather than the 10 we examine, and the phase values are omitted). The largest amplitude lines in $x$ and $y$ show several coincidences in their frequencies, revealing a SAT. Nevertheless, we notice that, for both coordinates, the line with the second largest amplitude has a frequency larger (in absolute values) than the one with the largest amplitude; besides, again for both coordinates, the largest and the 
Table I. Results of the frequency analysis of orbit 2768 in the non-rotating system.

\begin{tabular}{lrrrrr}
\hline$f_{x}$ & $A_{x}$ & $f_{y}$ & $A_{y}$ & $f_{z}$ & $A_{z}$ \\
\hline-0.12068913 & 0.898 & -0.12068925 & 1.214 & -0.04639543 & 0.119 \\
-0.36206809 & 0.794 & -0.36206796 & 0.964 & +0.04639558 & 0.109 \\
+0.12068937 & 0.704 & +0.12068926 & 0.953 & -0.43636165 & 0.081 \\
+0.36206772 & 0.372 & +0.36206795 & 0.452 & -0.19498364 & 0.046 \\
-0.84482556 & 0.247 & -0.84482518 & 0.301 & -0.28777377 & 0.041 \\
-0.08368269 & 0.180 & -1.32758238 & 0.172 & -0.91911898 & 0.033 \\
\hline
\end{tabular}

second largest amplitudes are of comparable size. Therefore, in this case, we take $f_{x}=0.36206809, f_{y}=0.36206796$ (i.e., they are equal within our $2 \times 10^{-4}$ precision limit). We see that the $z$ line with the largest amplitude (as well as its positive frequency counterpart) has a frequency lower than the one selected for $f_{x}$ and $f_{y}$ (or, for that matter, than the one corresponding to the largest amplitude in those coordinates). We notice, however, that the line with the third largest $f_{z}$ has a frequency $(-0.43636165)$ that has a symmetric counterpart, relative to the $f_{x}$ selected frequency $(-0.36206809)$, in the line with the fifth largest $f_{z}$ amplitude $\left(f_{z}=-0.28777377\right)$; again, the amplitudes of these two symmetric lines are of comparable size. Therefore, we choose $f_{z}=0.43636165$.

The importance of taking this extra care to select the fundamental frequencies, rather than taking just those corresponding to the largest amplitudes can be appreciated from Figure 1: we have plotted there the frequencies of maximum amplitude, together with those selected as indicated below, as a function of the reduced energy, $E / W_{\mathrm{o}}$ (i.e., the orbital energy divided by the potential at the center of the system); for clarity, only the low reduced energy section is shown because, as already indicated, it is there that the described effects are present. Orbit 2768, whose frequency analysis was discussed above, is the one at a reduced energy of 0.0396 . We notice that the four SATs with lowest reduced energy values lie well below the extrapolations of the frequency vs. reduced energy correlations in the three plots; conversely, they fit nicely on those extrapolations when their frequencies are derived with our recipe. The improvement is even clearer for the $y$ and $z$ frequencies of the boxes and boxlets (BBLs hereafter) because in those plots, besides having a larger number of cases, the extrapolation is only needed for the orbits with the lowest reduced energies, while there are also intermediate energy regions where coexist orbits that do need our 

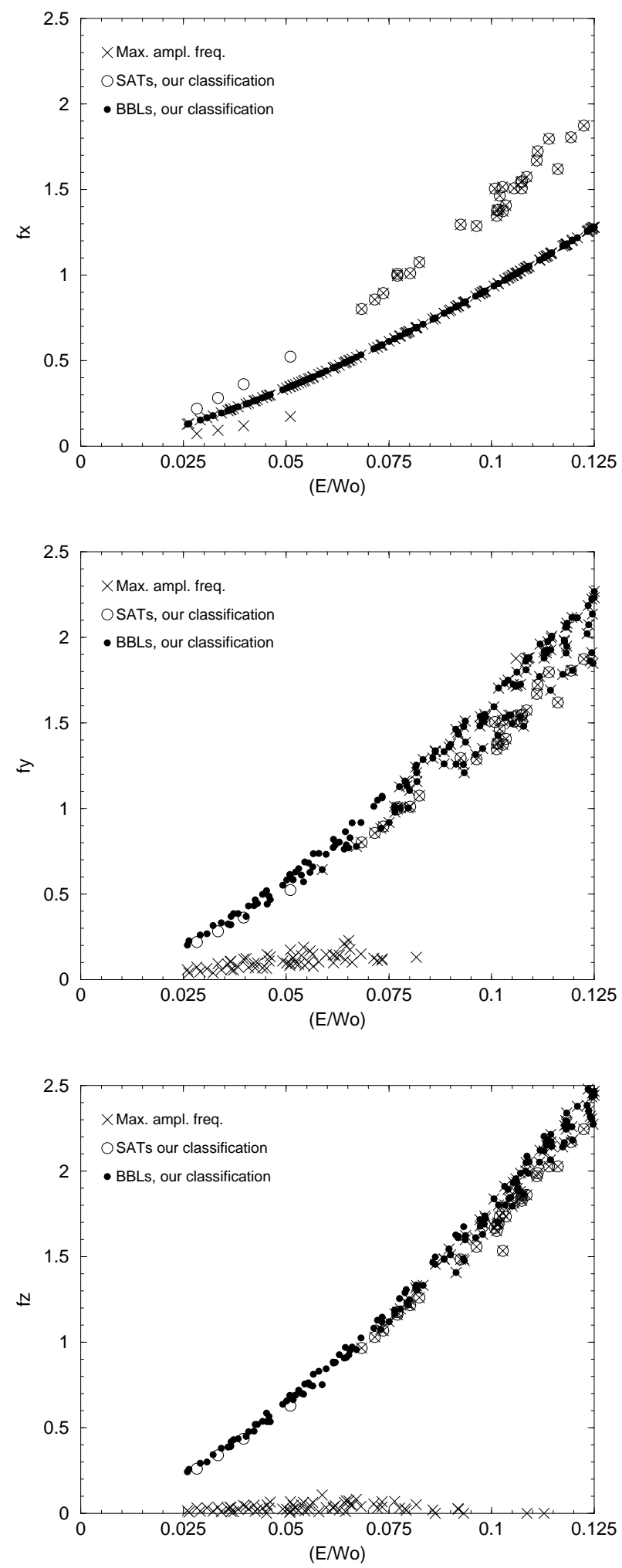

Figure 1. Frequencies of the maximum amplitude components and frequencies selected with our method $\left(f_{x}, f_{y}\right.$ and $f_{z}$, upper, middle and lower sections, respectively) vs. the reduced energy. 
treatment with those for which the largest amplitude frequencies are all right.

Our method can be summarized as follows. We work directly with the complex Fourier transform, just as the previous authors did, and we consider the negative frequencies only: if $f$ is the absolute value of the frequency and $A_{-}$and $A_{+}$the amplitudes corresponding, respectively, to the negative and positive values of the frequency, then $A_{-}=A_{+}(1+$ $f) /(1-f)$ or $A_{-}=A_{+}(1+f) /(f-1)$, depending on whether $f<1$ or $f>1$, i.e., it is always $A_{-}>A_{+}$.

We begin looking for the long axis tubes (LATs hereafter) and, for that purpose, we compare the maximum amplitudes of the $y$ and $z$ variables and select the largest one (say, $y$ ). Then, we compare its frequency with the three negative frequencies with the largest amplitudes of the other variable ( $z$ in this case): if there is a coincidence, the orbit is a LAT. We then check whether the second largest amplitude of the first variable ( $y$ in our example) has a frequency that is more negative than the one of the largest frequency and, at the same time, its amplitude is at least $70 \%$ of the latter; if that is so, we adopt the most negative frequency as the fundamental one of the first variable and we search among the frequencies of the second variable ( $z$ in this case) for the one which is equal to it, in order to adopt it as the fundamental frequency of the second variable. Finally, we take the frequency corresponding to the largest $x$ amplitude as $f_{x}$; all our LATs satisfed the relation $f_{x}<f_{y}=f_{z}$ (our code checks that) so that no additional operations were found necessary for them.

We then look for the SATs, beginning with the same method described for the LATs, except that now the $x$ and $y$ (rather than $y$ and $z$ ) variables are investigated. Nevertheless, in these cases we frequently found that the $f_{x}=f_{y}$ thus obtained were larger than the frequency (in absolute value) of the largest amplitude $z$ line; therefore, in those cases, the $f_{z}$ frequency is determined finding the couple of $z$ lines that are symmetric with respect to $f_{x}$ and adopting the largest (in absolute value) frequency of the pair as $f_{z}$.

For the orbits that are neither LATs nor SATs, we begin selecting $f_{x}$ as the frequency of the largest amplitude $x$ line. If the frequency of the largest amplitude $y$ line is larger than the former (in absolute value), this frequency is taken as $f_{y}$; otherwise, $f_{y}$ is determined from the pair of lines symmetric with respect to $f_{x}$, as explained before. Finally, $f_{z}$ is obtained in the same way as $f_{y}$.

All the regular orbits in the non-rotating and rotating systems could be classified with this method. The frequency ratios so obtained allow us to make an initial, rather coarse, classification of our regular orbits, more or less along the lines of that of Kalapotharakos and Voglis (2005), 
Table II. Number of different types of orbits in both systems.

\begin{tabular}{lrrrrrr}
\hline Rotating/Non-rot. & LAT & SAT & BBL & Part. Ch. & Fully Ch. & Total \\
\hline LAT & 49 & 0 & 0 & 2 & 1 & 52 \\
SAT & 0 & 241 & 0 & 22 & 6 & 269 \\
BBL & 0 & 0 & 1129 & 38 & 18 & 1185 \\
Part. Ch. & 3 & 23 & 119 & 144 & 38 & 327 \\
Fully Ch. & 5 & 9 & 66 & 102 & 1457 & 1639 \\
Total & 57 & 273 & 1314 & 308 & 1520 & 3472 \\
\hline
\end{tabular}

recognizing: 1) LATs, with $f_{y} / f_{z}=1$; 2) SATs with $\left.f_{x} / f_{z}=f_{y} / f_{z} ; 3\right)$ Boxes and boxlets (BBL) as all the other regular orbits. Inner and outer long axis tubes might be also distinguished, because the former have lower $f_{x} / f_{z}$ values than the latter but, as there were only 2 outer long axis tubes in our non-rotating system and none in our rotating one, we felt unnecessary to draw this distinction. We preferred to keep the older name "boxlet", rather than "higher order resonant tube" used by Kalapotharakos and Voglis, because these orbits appear on the same region of the frequency map as the boxes and their distribution in configuration space is similar to that of the boxes (see, e.g., Figs. 7 and 8 of Muzzio et al., 2005).

A more refined classification was performed later, selecting the most important resonances from the frequency map and separating the boxlets according to the resonances that they obey.

\section{Results}

Throughout the present paper, the errors of the percentages are the dispersions derived from the binomial distribution, i.e., for a percentage $p$, obtained from a total number of data $N$, the dispersion $\sigma$ is:

$$
\sigma=\sqrt{p(100-p) / N}
$$

Table II presents the results of the orbit classification for both systems. We see that $47.35 \pm 0.85 \%$ of the orbits in the non-rotating system, and $43.38 \pm 0.84 \%$ of those in the rotating system were regular; the difference between these percentages amounts to 3.3 its dispersion, so that it is highly significant. Alternatively, the percentages of regular orbits found in the non-rotating system with the parameters obtained from the fittings at $0-10-20 T_{\text {cr }}$ and $0-200-400 T_{\text {cr }}$ were $46.72 \pm 0.85$ and $46.57 \pm 0.85$, respectively, so that they are essentially 
the same as those found with the parameters of the fitting at $0 T_{\mathrm{cr}}$. Voglis, Kalapotharakos and Stavropoulos (2002) also checked how the fraction of chaotic orbits was altered when the potential was fitted to the $\mathrm{N}$-body distribution at different times and they found that "less than $3 \%$ " of their orbits had changed the character of their motion. They do not give the error of that figure but, considering that they used a number of particles of the same order as the one we used, that error is probably similar to ours and their fraction is thus not significantly different from those we found (i.e., about $0.7 \% \pm 1.2 \%$ ).

Since the differences beween the potentials of the non-rotating and rotating systems are strongly dependent on radius, we investigated whether the fractions of regular orbits showed some dependence with the orbital energy as follows. We chose different values of the reduced energy $(0.100,0.319$ and 0.500$)$ to divide each sample of 3,472 orbits in two sets and we found the percentages of regular orbits in each set. Nevertheless, no dependence of chaoticity with the orbital energy was found because, just as it happened with the whole samples, the nonrotating system turned out to have about $4 \%$ more regular orbits than the rotating one on both sides of the three energy values considered: e.g., $52.30 \pm 1.20$ vs. $48.62 \pm 1.20$ for $E / W_{\mathrm{o}}>0.319$ (the median value of the reduced energy) and $42.40 \pm 1.19$ vs. $38.13 \pm 1.17$ for $E / W_{\mathrm{o}}<0.319$, respectively.

Nevertheless, we should recall that in rotating systems the forces change not only by the addition of the centrifugal force (that affects the potential), but also by the Coriolis force (that leaves it unchanged) and the relevance of the latter to the onset of chaos in galactic satellites has been shown by Muzzio (2001) and Carpintero et al. (2003). Those authors found that the artificial suppresion of the Coriolis force drastically reduced the amount of chaos in those systems and, besides, that the whole range of orbital energies was significantly affected. Therefore, we also artificially suppressed the terms of the Coriolis force in the LIAMAG routine and found the corresponding Lyapunov exponents of the 3,472 orbits in the rotating system: it turned out that $47.84 \pm 0.85$ were then classified as regular, that is, essentially the same percentage of regular orbits found in the non-rotating system.

Among the regular orbits, the percentages of LAT, SAT and BBL orbits are, respectively, $3.47 \pm 0.45 \%, 16.61 \pm 0.92 \%$ and $79.93 \pm 0.99 \%$ in the non-rotating system, and $3.45 \pm 0.47 \%, 17.86 \pm 0.99 \%$ and $78.69 \pm 1.06 \%$ in the rotating one; all the differences between the percentages of the same kind of orbits in the two systems are smaller than the corresponding dispersions, so that they are not significant. Finally, among the chaotic orbits in the non-rotating system $16.85 \pm 0.88 \%$ were partially chaotic and $83.15 \pm 0.88 \%$ fully chaotic, while the correspond- 
Table III. Percentages of the main resonant orbits in both systems.

\begin{tabular}{lrr}
\hline Resonance & Non-rot. system & Rotating system \\
\hline$(2,0,-1)$ & $2.37 \pm 0.38 \%$ & $2.66 \pm 0.41 \%$ \\
$(3,-2,0)$ & $4.87 \pm 0.53 \%$ & $4.18 \pm 0.52 \%$ \\
$(2,1,-2)$ & $4.32 \pm 0.50 \%$ & $3.92 \pm 0.50 \%$ \\
$(3,-3,1)$ & $1.76 \pm 0.32 \%$ & $1.59 \pm 0.32 \%$ \\
$(5,-3,0)$ & $5.17 \pm 0.55 \%$ & $5.51 \pm 0.51 \%$ \\
\hline
\end{tabular}

ing figures for the rotating system are $16.63 \pm 0.84 \%$ and $83.37 \pm 0.84 \%$; the differences between the percentages of partially (or fully) chaotic orbits in each system are thus not significant, as they amount to only 0.18 of the corresponding dispersion.

Figures 2 and 3 present the frequency maps, respectively, for the non-rotating and the rotating systems. No significant differences are apparent between them; the most obvious, albeit minor, one probably being the couple of outer LATs that are present in the non-rotating system (near $f_{y} / f_{z}=1.00, f_{x} / f_{z} \simeq 0.85$ ) and not in the rotating one.

Resonances $(2,0,-1),(3,-2,0),(2,1,-2),(3,-3,1)$ and $(5,-3,0)$ stand out very clearly in both Figures; the presence of several others can be surmised but, at any rate, they would comprise very small fractions of orbits. Table III gives the percentages of orbits in each of the main resonances, with respect to the total numbers of regular orbits, separately for the non-rotating and rotating systems. Again, there are no significant differences between the results for the two systems.

\section{Discussion}

The method used here to classify the regular orbits, following the footsteps of Papaphilippou and Laskar (1998) and of Kalapotharakos and Voglis (2005), seems to be preferable to the fully automatic one of Carpintero and Aguilar (1998). Here we classified all the regular orbits, while $15.3 \%$ of the orbits in the non-rotating system could not be classified by Muzzio et al. (2005) with the latter method. Although, later on, Carpintero (private communication) succeeded in classifying all those orbits after relaxing the criteria used to check for unresolvable lines, it seems advisable to obtain first the frequency map and detect the main resonances there, rather than letting the computer automatically search for them. 

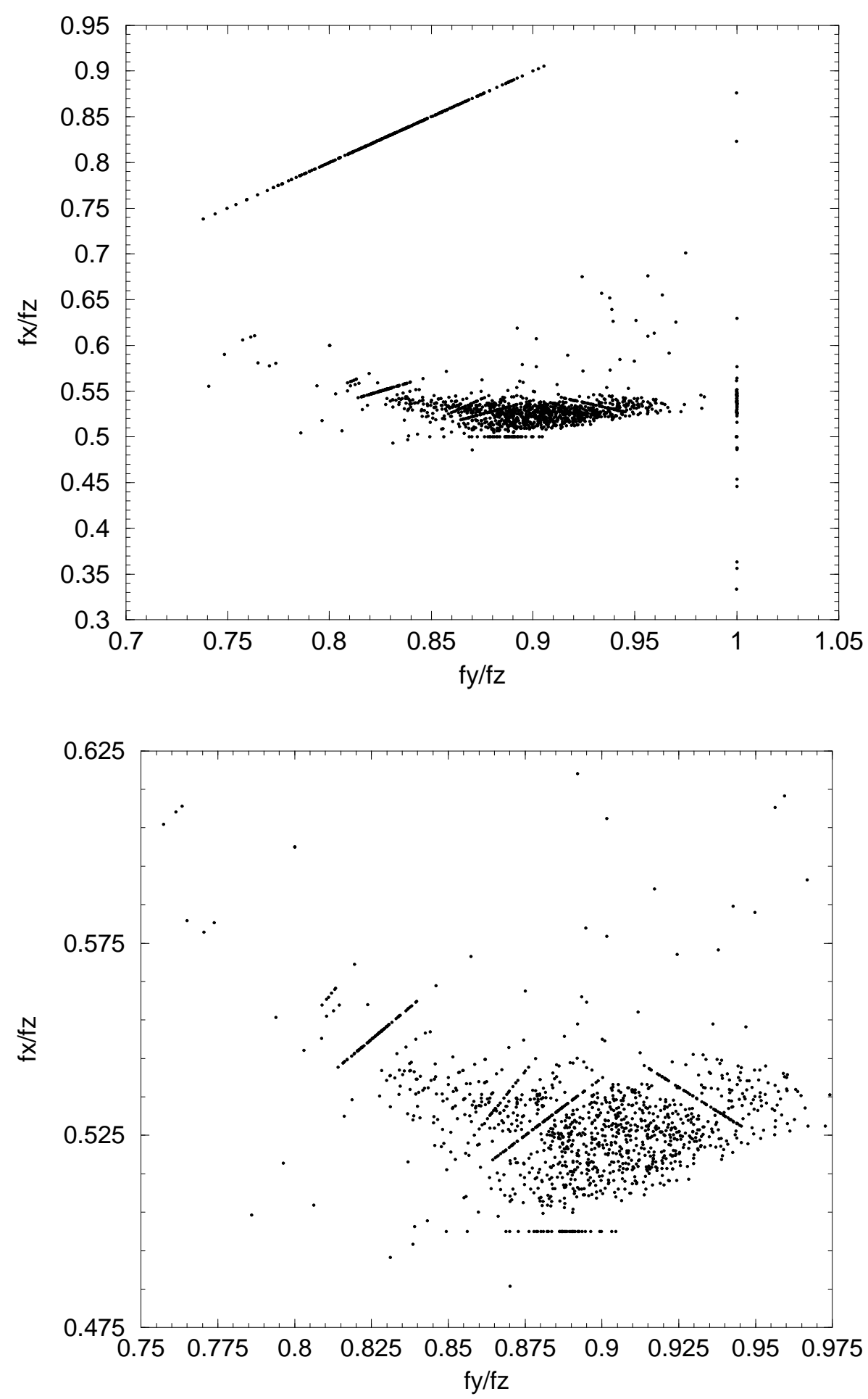

Figure 2. The whole frequency map for the non-rotating system (upper part) and an enlargement of the region corresponding to boxes and boxlets (lower part) 

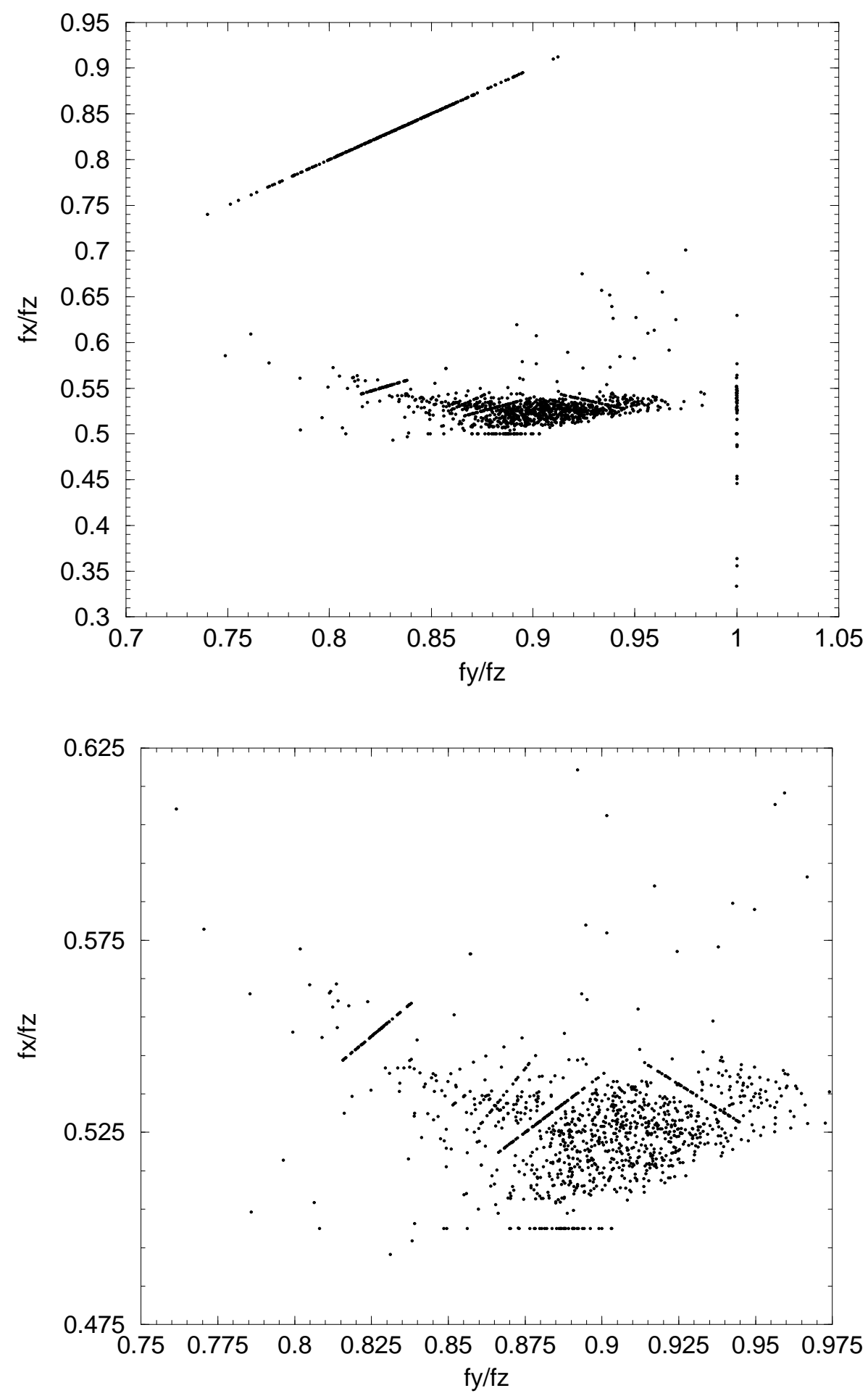

Figure 3. Same as Fig. 2 for the rotating system 
Our results indicate that the only significant difference in orbital structure between the non-rotating and rotating system is that the latter has a slightly larger fraction of chaotic orbits. This is a reasonable result, because the rotation causes a break of symmetry that may increase the presence of chaos, but it is interesting that even a rotation as slow as the one considered here can give rise to a statistically significant increase.

That break of symmetry is probably one of the reasons why, with a smaller average change in the potential, rotation causes a larger effect on the fraction of chaotic orbits than do the changes that arise from fitting the potential to the $\mathrm{N}$-body distribution at different times. The effect of the Coriolis force is probably the best explanation for the significant increase of chaos in the rotating system (relative to the non-rotating one) and for the fact that such increase shows no significant correlation with the orbital energy, as one might expect if the centrifugal force was the only one involved.

Neither the relative number of partially to fully chaotic orbits nor the relative numbers of the different classes of regular orbits showed any significant dependance on rotation, probably because it is so slow.

It is worth mentioning that in the case of the real galaxy NGC 4365 Statler et al. (2004) found that it was seemingly safe to conclude that figure rotation was dynamically unimportant in the observable part of the system.

\section{Acknowledgements}

The comments of an anonymous referee were very useful to improve the original version of the present paper and are greatfully acknowledged. We are very grateful to Sverre J. Aarseth, Luis A. Aguilar, David Nesvorný and Daniel Pfenniger for allowing us to use their codes, and to Ruben E. Martínez for technical assistance. This work was supported with grants from the Consejo Nacional de Investigaciones Científicas y Técnicas de la República Argentina, the Agencia Nacional de Promoción Científica y Tecnológica and the Universidad Nacional de La Plata.

\section{References}

Aarseth, S. J.: 2003, Gravitational N-body simulations, Cambridge University Press Binney, J., Spergel, D.: 1982, 'Spectral stellar dynamics', Astrophys. J., Vol. 252, pp. 308-321 
Binney, J., Spergel, D.: 1984, 'Spectral stellar dynamics. II- The action integrals', $M N R A S$, Vol. 206, pp. 159-177

Binney, J., Tremaine, S.: 1987, Galactic Dynamics, Princeton University Press

Carpintero, D. D., Aguilar, L. A.: 1998, 'Orbit classification in arbitrary 2D and 3D potentials', MNRAS, Vol. 298(1), pp. 1-21

Carpintero, D. D., Muzzio, J. C., Vergne, M. M., Wachlin, F. C.: 2003, 'Chaotic orbits in galactic satellites', Celest. Mech. and Dynam. Astron., Vol. 85(247267),

Cruz, F., Aguilar, L.A. and Carpintero, D.D.: 2002, 'A new method to find the potential center of N-body systems', Rev. Mexicana Astron. Astrof., Vol. 38, pp. $225-231$

Hernquist, L. and Barnes, J.: 1990, 'Are some N-body algorithms intrinsically less collisional than others?', Astrophys. J., Vol. 349, pp. 562-569

Kalapotharakos C. and Voglis N.: 2005 'Global dynamics in self-consistent models of elliptical galaxies' Celest. Mech. Dynam. Astron., Vol. 92(1-3), 157-188

Merritt, D.: 1999, 'Elliptical galaxy dynamics', Publ. Astron. Soc. Pacific, Vol. 111(756), pp. 129-168

Muzzio, J.C.: 2001, 'Chaos in a simple dynamical system', Anales Acad. Nac. de Cs. Ex., Fís. y Nat., Vol. 53, 65-72

Muzzio, J.C.: 2003, 'Chaos in elliptical galaxies', Bol. Asoc. Argentina Astron., No. 45, 69

Muzzio, J.C., Carpintero, D.D. and Wachlin, F.C.: 2005, 'Spatial structure of regular and chaotic orbits in a self-consistent triaxial stellar system', Celest. Mech. and Dynam. Astron., Vol. 91(1-2), 173-190

Muzzio, J.C. and Mosquera, M.E.: 2004, 'Spatial structure of regular and chaotic orbits in self-consistent models of galactic satellites', Celest. Mech. and Dynam. Astron., Vol. 88(4), pp. 379-396

Papaphilippou, Y. and Laskar, J.: 1998, 'Global dynamics of triaxial galactic models through frequency map analysis', Astron. \& Astrophys., Vol. 329, pp. 451-481

Schwarzschild, M.: 1979 'A numerical model for a triaxal stellar system in dynamical equilibrium', ApJ, 232, pp. 236-247

Šdlichovský, M. and Nesvorný, D.: 1997 'Frequency modified Fourier transform and its application to asteroids', Celest. Mech. Dynam. Astron., 65, pp. 137-148

Sparke, L.S. and Sellwood, J.A.: 1987 'Dissection of an N-body bar' $M N R A S$, Vol. 225, pp. 653-675

Statler, T.S., Emsellem, E., Peletier, R.F. and Bacon, R.: 2004 'Long-lived triaxiality in the dynamically old elliptical galaxy NGC 4365: a limit on chaos and black hole mass' MNRAS, Vol. 353, pp. 1-14

Udry, S. and Pfenniger, D.: 1988 'Stochasticity in elliptical galaxies' Astron. Astroph., Vol. 198(1-2), pp. 135-149

Voglis N., Kalapotharakos C. and Stavropoulos I.: 2002, 'Mass components in ordered and in chaotic motion in galactic $N$-body models' $M N R A S$, Vol. 337(2), 619-630

Wachlin, F.C. and Ferraz-Mello, S.: 1998, 'Frequency map analysis of the orbital structure in elliptical galaxies' $M N R A S$, Vol. 298(1), 22-32

White, S.D.M.: 1983, 'Simulations of sinking satellites', Astroph. J., Vol. 274, pp. 53-61 